\title{
Stress-Induced Failure Modes in High-Tuning Range RF MEMS Varactors
}

\author{
Trushal Chokshi and Dimitrios Peroulis \\ School of Electrical and Computer Engineering, and Birck Nanotechnology Center \\ Purdue University, West Lafayette, USA \\ tchokshi@purdue.edu,dperouli@purdue.edu
}

\begin{abstract}
In this paper we focus on electromechanical modeling of high-tuning range MEMS varactors with a focus on failures caused by residual compressive stress. In particular, we quantitatively evaluate for the first time a high-tuning range parallel-plate MEMS varactor in the presence of residual compressive stress. A 3D model generated in ANSYS agrees very favorably with the measured data and explains non-ideal discontinuities in the varactor's $C-V$ curve. It is interesting to note that, although the failures considered in this paper are not encountered in RF MEMS switches, they become particularly important in analog MEMS varactors since they directly impact their effective tuning range.
\end{abstract}

\section{Introduction AND Motivation}

RF MEMS switches are currently considered among the most promising tunable devices due to their excellent RF performance and virtually zero power consumption [1]. Despite their advantages, however, a number of serious reliability and cost issues remain unanswered, including contact area damage and wear [2], power handling [3] and dielectric charging [4].

It is interesting to note that these reliability and packaging concerns in today's MEMS switching technology are directly linked to their designed mode of operation that relies on some type of direct metal-to-metal [5] or metalto-dielectric contact [6]. Other MEMS devices that operate in completely contact-less modes, such as the Analog Devices accelerometers and the Agilent Film Bulk Acoustic Resonator (FBAR) filters, do not exhibit these difficulties, have already become commercially available and are massively produced. Although their design and complexity are comparable to MEMS switches, their crucial advantage lies on the lack of any type of contact.

Motivated by these devices, several researchers have focused on contact-less devices and primarily high tuningrange varactors. The authors, for instance, have already demonstrated completely contact-less millimeterwave MEMS variable capacitors with tuning range of up to $4: 1$ [7] and have discussed reliability issues related to creep and packaging [8]. Unlike MEMS switches, these capacitors are immune to stiction and do not suffer from dielectric charging since no thin dielectric films are necessary for their operation. However, non-ideal $C-V$ curves are often observed in measurements of these varactors [7], [9]. It is the purpose of this paper to provide for the first time quantitative results about these non-ideal behavior and propose methods to alleviate these issues.

\section{Measured Non-Ideal $C-V$ Curves}

Figs. 1 and 2 show two examples of MEMS analog variable capacitors with non-ideal $C-V$ curves that have appeared in the literature and have not been explained yet [7], [9]. The varactor of Fig. 1 (we will call it varactor A) is

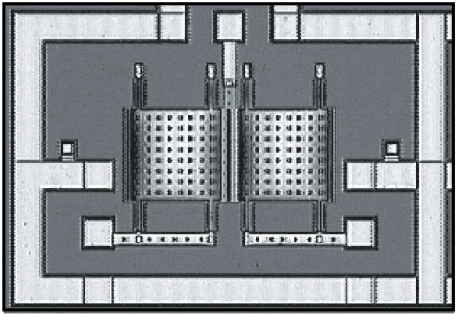

(a)

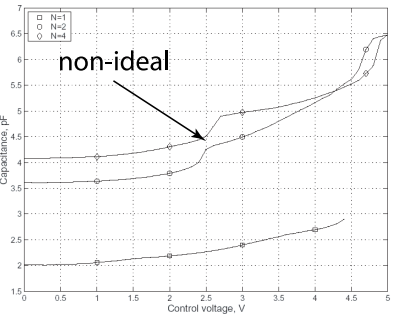

(b)
Fig. 1. (a) Differential analog MEMS varactor $(N=1$ finger for this picture) and (b) its $C-V$ curve for 1,2 and 4 fingers after [9].

a differential multi-finger parallel-plate device that is fabricated with the commercially available MUMPS process. On the other hand, the varactor of Fig. 2 (we will call it varactor B) is built in our solid-state laboratory with a custom fabrication process [7] and is based on the extended-tuning range approach. It is interesting to note that although these two varactors are based on very different geometries and fabrication processes, the discontinuity in their $C-V$ curve around 2.3 and $15 \mathrm{~V}$ is common in both of them. This is a very undesirable effect since it limits the effective tuning range of the device and contributes to unwanted non-linearity to the circuit. Therefore it is important to understand the cause of this effect so that the design can be improved.

\section{VARACTOR B OPERATION}

While our results can be utilized for both cases, this paper focuses on analyzing varactor B. Consequently, for completeness we summarize the basic operation of this varactor here. The design is based on two beams, namely the lower and upper beams. The lower beam is $1 \mu \mathrm{m}$ thick, is suspended approximately $2 \mu \mathrm{m}$ above the center conductor of a $60 / 100 / 60 \mu \mathrm{m}$ coplanar waveguide (cpw) and is anchored at its ground planes. The upper beam is $10 \mu \mathrm{m}$ thick and is connected only at the center of the lower beam through three $6-\mu \mathrm{m}$-thick vias. Consequently the upper beam is $8 \mu \mathrm{m}$ above the substrate. It also extends over two square pads that are used for actuating the device. These square pads are completely isolated from the RF line and are not connected to the cpw ground planes.

When an electrostatic potential is applied between the upper beam and the two actuation pads, an electrostatic force is felt by the upper beam. However, this beam does not deflect due to its very large spring constant. It forces though the lower beam to deflect. Because of the height difference between the two beams, the pull-in instability for the upper beam does not occur until it moves by approximately $2.5 \mu \mathrm{m}$ downwards. This means that the upper beam does not collapse before the lower beam touches the center conductor. As a result, the tuning range of the var- 


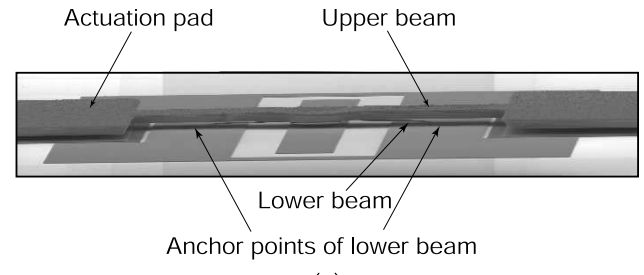

(a)

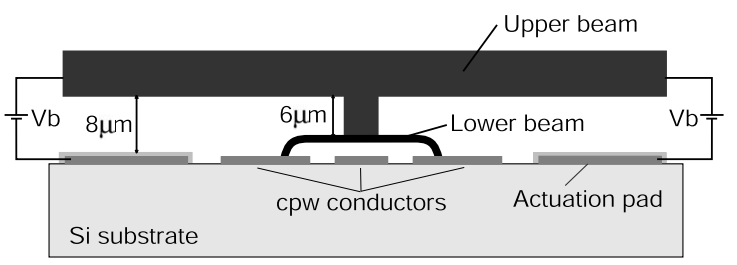

(b)

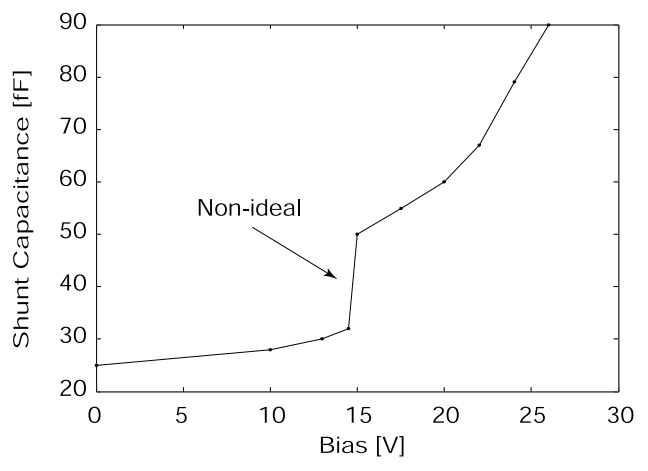

(c)

Fig. 2. (a) High-tuning range analog MEMS varactor, (b) a simple schematic of the device, and (c) its measured non-ideal $C-V$ curve (after [7].

actor can be designed to be (theoretically) arbitrarily high. In practice it is limited by the residual stress in the fabrication process as well as by the desired maximum allowable RF power.

In [7] a hypothesis is proposed to explain the discontinuous effect. It is assumed that it is due to the initial upward warping of the lower beam. When a bias voltage is applied the beam starts deflecting downwards which leads to compressive stress being built in the beam. It is hypothesized that the point of discontinuity in the $C-V$ curve is the point where the compressive stress exceeds a critical value and buckling occurs. The next section proves the correctness of this hypothesis.

\section{Modeling}

To explain the undesired discontinuous effect we model varactor B in ANSYS [10] using a 3D electromechanical approach. The generated model is shown in Fig. 3. The geometry of this model corresponds exactly to the geometry of the real structure. This includes the undesired upward curling of the lower beam that is observed in the real structure. From RF measurements similar to the ones shown in Fig. 2c it was extracted that the final height of the lower beam is approximately $4 \mu \mathrm{m}$ instead of the intended $2 \mu \mathrm{m}$ as mentioned in the previous section. This effect was modeled in ANSYS by introducing an appropriate upward curvature in the lower beam (see Fig. 3).

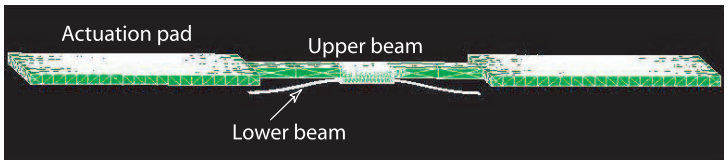

Fig. 3. ANSYS 3D electromechanical model that corresponds to varactor $\mathrm{B}$. The lower beam has been bent the same way as in the real structure. All the necessary parameters for the model are given in Table I.

TABLE I

Geometric And material PARAmeters of THE MOdEL SHOWN IN FIG. 3

\begin{tabular}{lcr} 
Parameter & Symbol & Value \\
\hline Lower beam length $[\mu \mathrm{m}]$ & $L_{1}$ & 300 \\
Lower beam width $[\mu \mathrm{m}]$ & $w_{1}$ & 20 \\
Lower beam thickness $[\mu \mathrm{m}]$ & $t_{1}$ & 1 \\
Beams' Young's modulus [GPa] & $\mathrm{E}$ & 80 \\
Beams' Poisson's ratio & $\nu$ & 0.42 \\
Upper beam length $[\mu \mathrm{m}]$ & $L_{2}$ & 920 \\
Upper beam width $[\mu \mathrm{m}]$ & $w_{2}$ & 100 \\
Upper beam thickness $[\mu \mathrm{m}]$ & $t_{2}$ & 13 \\
Actuation pad area $\left[\mu \mathrm{m}^{2}\right]$ & $A$ & $360 \times 360$ \\
Nominal air-gap (lower beam) $[\mu \mathrm{m}]$ & $g$ & 2 \\
True air gap (after curling) $[\mu \mathrm{m}]$ & $g_{0}$ & 4 \\
Beams' mesh elements & $N_{1}$ & 24,710 \\
Air-gap mesh elements & $N_{2}$ & 5437 \\
Substrate mesh elements & $N_{3}$ & 175
\end{tabular}

An infinitesimally small upward bent in the lower beam can initiate the desired amount of upward warping when subjected under compressive stress. Furthermore, by assigning a positive coefficient of thermal expansion to the lower beam and then subjecting it to a uniform temperature (above the set reference temperature) we can also create the necessary compressive stress. All of the relevant geometric and material parameters are summarized in Table I.

Unfortunately the model of Fig. 3 could not be directly used to predict the instability point or the post-buckling behavior of the beam. This was due to a slight tilt that was inevitably introduced in the upper beam when the compressive stress in the lower beam approached its buckling stress value. The amount of tilt increased at every subsequent step of the simulation until the upper beam (not the lower one) touched the substrate.

In order to avoid this behavior we had to simplify the structure and remove the upper beam, thus simulating only the lower beam. To compensate for this effect, we apply an equivalent mechanical pressure on the region of the lower beam where the upper beam were attached. This mechanical pressure equals the pressure generated by the electrostatic force on the upper beam's actuation pads. The equivalent mechanical pressure can be easily calculated (including the fringing field capacitance) and thus displacement vs. voltage curves for these varactors can be generated after the buckling point. The details of this analysis will appear in an appropriate journal. 


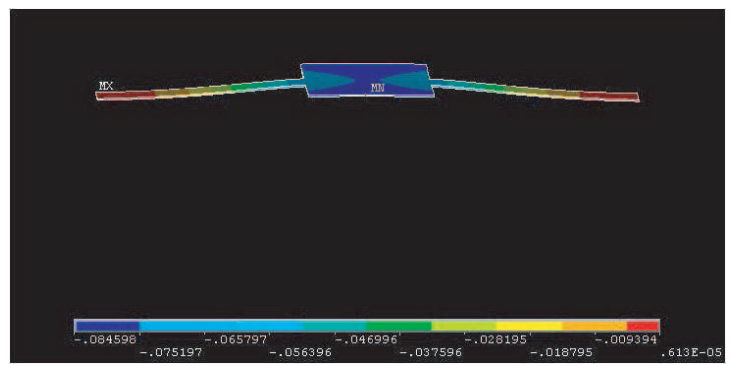

(a)

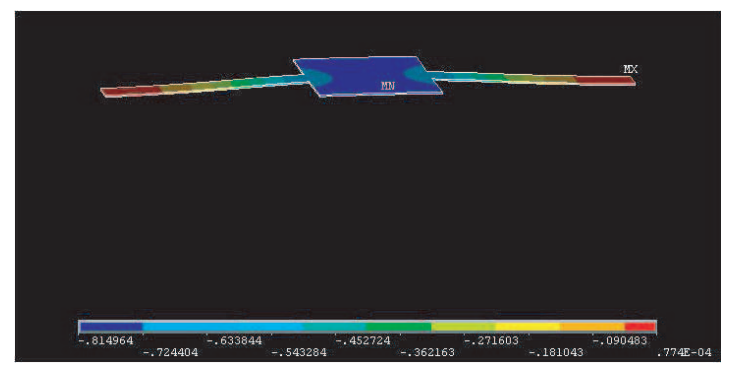

(b)

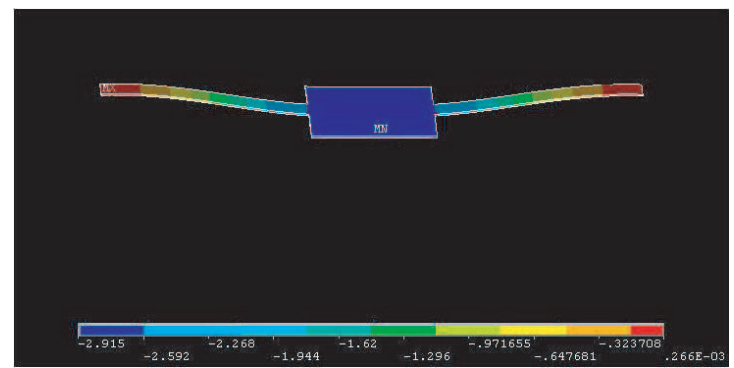

(c)

Fig. 4. A 300- $\mu \mathrm{m}$ lower beam at three different stages: (a) initial state $\left(g=4 \mu \mathrm{m}, V_{b}=0 V\right)$, (b) pre-buckling state $(g=3.2 \mu \mathrm{m}$, $\left.V_{b}=14.4 \mathrm{~V}\right)$, (c) post-buckling state $\left(g=1 \mu \mathrm{m}, V_{b}=15.9 \mathrm{~V}\right)$.

Fig. 4 shows the simulated lower beam at three steps: (i) initial condition $\left(V_{b}=0 \mathrm{~V}\right)$ where the beam is deflected $4 \mu \mathrm{m}$ above the substrate, (ii) just before buckling ( $V_{b}=$ $14.4 \mathrm{~V}$ ) where the structure is $3.2 \mu \mathrm{m}$ above the substrate, and (iii) just after buckling $\left(V_{b}=15.9 \mathrm{~V}\right)$ where the distance between the lower beam and the substrate is approximately $1 \mu \mathrm{m}$. Fig. 7 shows the complete simulated deflection-voltage curve of this beam.

Particular attention has to be paid, however, on the fact that although we have assumed a uniform thickness of the lower beam, this is not the case due to the connection of the lower and upper beams (Fig. 2). This connection takes place over an area of $80 \times 100 \mu \mathrm{m}^{2}$. In other words, the thickness of the lower beam in the proposed model has to be increased from 1 to $10 \mu \mathrm{m}$ over this aforementioned area. Since this is a significantly stiffer section $(11 \mu \mathrm{m}$ thick versus $1 \mu \mathrm{m}$ for the rest of the lower beam), in a first order buckling analysis we can substitute the actual lower beam with an effective lower beam whose length would be reduced by $80 \mu \mathrm{m}$. This is explained graphically in Fig. 5 .

The simulated deflection-voltage curve of the $220-\mu \mathrm{m}$ beam is shown in Fig. 6. The same graph presents the extracted deflection-voltage curve based on the measurements shown in Fig. 2. Despite the approximations, the
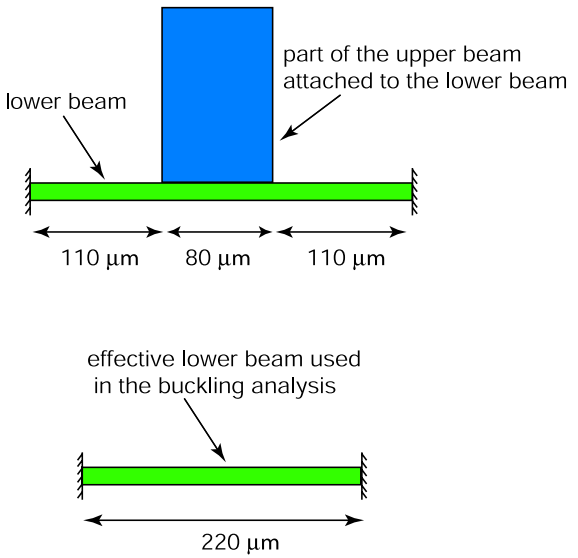

Fig. 5. Actual and effective lower beams used in the buckling analysis. Due to the stiffness of the center section of the lower beam, we replace it with an effective beam with a uniform thickness but reduced length.

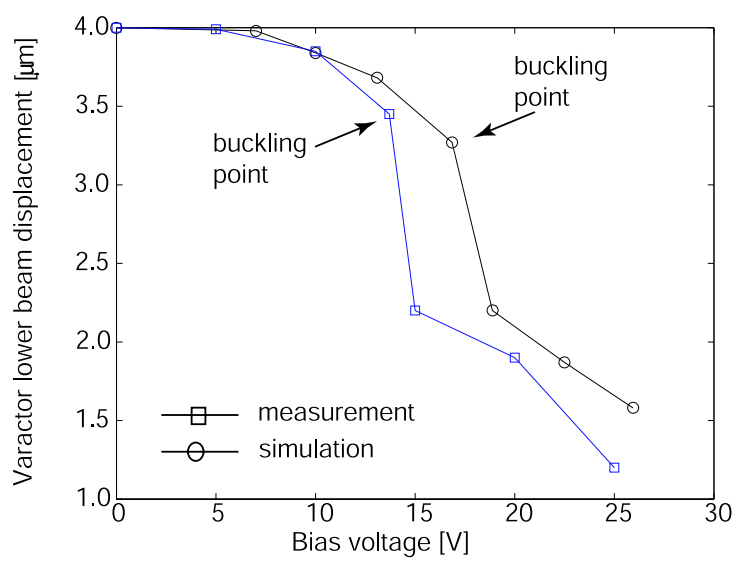

Fig. 6. Simulated and measured deflection-voltage curves for MEMS varactor $\mathrm{B}$. The buckling occurs at $13.7 \mathrm{~V}$ (measurements) and at $16.8 \mathrm{~V}$ (simulations).

complexity of the structure and the non-linear nature of buckling, reasonably good agreement is observed between simulated and measured data. In the actual experiment buckling occurred at $13.7 \mathrm{~V}$, while the simulated buckling point is at $16.8 \mathrm{~V}$.

\section{Structural improvements}

It is interesting to note that the $C-V$ discontinuity can be removed not only by reducing the in-plane compressive stress, but also by changing the design of the lower beam. In this work we consider the simple case of reducing the length of the lower beam. Shorter beams can sustain higher levels of compressive stress before buckling. Consequently, they are more robust and less sensitive to process fluctuations. The penalty paid is an increased tuning voltage.

To illustrate this point, we first find out the amount of initial compressive stress that would produce a curvature of about $2 \mu \mathrm{m}$ in a beam of length $300 \mu \mathrm{m}$. This stress was numerically evaluated to be $73.1 \mathrm{MPa}$. When incorporated in a $160-\mu \mathrm{m}$ beam, it results in a curvature of about 1.1 $\mu \mathrm{m}$. Fig. 7 shows the deflection-voltage curve for a $160-\mu \mathrm{m}$ beam and compares it with a $300-\mu \mathrm{m}$ beam. For the given 


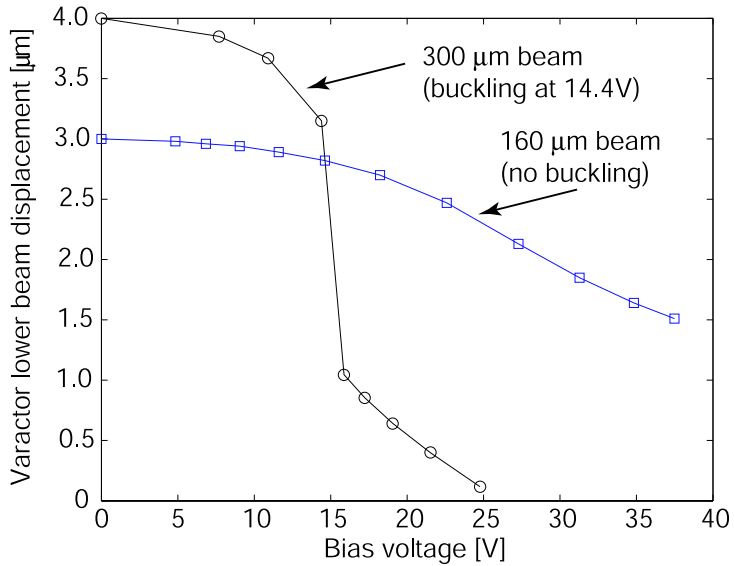

Fig. 7. Simulated deflection-voltage curves for $300-$ and $160-\mu \mathrm{m}$ lower beams. The initial compressive stress $(73.1 \mathrm{MPa})$ is the same for both beams. Unlike the $300-\mu \mathrm{m}$ beam, no discontinuity is observed for the $160-\mu \mathrm{m}$ beam.

compressive stress of $73.1 \mathrm{MPa}$, the $160-\mu \mathrm{m}$ beam does not suffer any discontinuity.

These results prove that the discontinuous behavior of the $C-V$ curve is directly related to the initial upward curling of the lower beam that is caused by residual compressive stress. Consequently, this issue is not an inherent disadvantage of the varactor geometry and can be alleviated even in the presence of a residual compressive stress by appropriately altering the lower beam design.

\section{Vi. Conclusion}

This paper has focused on 3D electromechanical modeling of analog MEMS varactors. The presented models, which are verified by measurements, clearly explain nonideal $C-V$ curves commonly encountered in this type of devices. The results of this paper directly link for the first time the discontinuous $C-V$ curves with residual compressive stress. Consequently, the discussed techniques can be used as a useful tool to aid the MEMS engineer in the design of variable capacitors in the presence of residual stress.

\section{ACKNOWLEDGMENT}

This work has been supported by the Multifunctional Adaptive Radio, Radar and Sensors (MARRS) MURI Project (Award Reference Number 2001-0694-02) and the Collaborative Technology Alliances in Advanced Sensors Program sponsored by the U.S. Army Research Laboratory (contract DAAD-19-01-2-0008).

\section{REFERENCES}

[1] G. M. Rebeiz, "RF MEMS, theory, design and technology" John Wiley and Sons, 2003.

[2] D. Hyman and M. Mehregany, "Contact physics of gold microcontacts for MEMS switches," IEEE Trans. on Components and Packaging Technologies, vol. 22, pp. 357-364, September 1999.

[3] J. Wellman and A. Garcia, "High Power ( $>1 \mathrm{~W})$ application of RF MEMS Lifetime Performance Evaluation", Jet Propulsion Laboratory evaluation report, January, 2003 (available online at: http://nepp.nasa.gov/DocUploads/5FAB12BE-15FE-4A30BA522BC13547FE60/RFMEMSX.pdf)

[4] X. Yuan, S. Cherepko, J. Hwang, C.L. Goldsmith, C. Nordqusit and C. Dyck "Initial observation and analysis of dielectriccharging effects on RF MEMS capacitive switches," 2004 IEEE MTT-S Int. Microwave Symp. Dig., vol. 3, pp. 1943-1946, June 2004 .
[5] R.E. Mihailovich, M. Kim, J.B. Hacker, E.A. Sovero, J. Studer, J.A. Higgins and J.f. DeNatale, "MEM relay for reconfigurable RF circuits," IEEE Microwave and Wireless Components Letters, vol. 11, pp. 53-55, Feb. 2001.

6] D. Peroulis, S. P. Pacheco, K. Sarabandi and L. P. B. Katehi, "Electromechanical considerations in developing low-voltage RF MEMS switches," IEEE Trans. Microwave Theory $\&$ Tech., vol. 51, no. 1, pp. 259-270, January 2003.

[7] D. Peroulis and L. P. B. Katehi, "Electrostatically-tunable analog RF MEMS varactors with measured capacitance range of $300 \%$," 2003 IEEE MTT-S Int. Microwave Symp. Dig., vol. 3, pp. 17931796, June 2003.

[8] D Peroulis, Yumin Lu and L. P B Katehi, " Highly reliable analog MEMS varactors," 2004 IEEE MTT-S Int. Microwave Symp. Dig., vol. 2, pp. 869-872, June 2004.

9] G. V. Ionis, A. Dec and K. Suyama, "Differential Multi-Finger MEMS Tunable Capacitors for RF Ingebgrated Circuits", 2002 IEEE MTT-S Int. Microwave Symp. Dig. vol. 1, pp. 345-348, June 2002.

[10] ANSYS Corporation, http://www.ansys.com, ANSYS version 8.0. 